International Journal of Algebra, Vol. 8, 2014, no. 4, 195 - 204

HIKARI Ltd, www.m-hikari.com

http://dx.doi.org/10.12988/ija.2014.4214

\title{
Induced Representations of Hopf Algebras
}

\author{
Ibrahim Saleh \\ Mathematics Department \\ University of Wisconsin-Marathon \\ Wausau, WI 54401, USA
}

Copyright (c) 2014 Ibrahim Saleh. This is an open access article distributed under the Creative Commons Attribution License, which permits unrestricted use, distribution, and reproduction in any medium, provided the original work is properly cited.

\begin{abstract}
Hopf representation is a module and comodule with a consistency condition that is more general than the consistency condition of Hopf modules. For a Hopf algebra $H$, we construct an induced Hopf representation from a representation of a bialgebra $B$ using a bialgebra epimorphism $\pi: H \rightarrow B$. Application on the quantum group $E_{q}(2)$ is given.
\end{abstract}

Mathematics Subject Classification: 16T05, 06B15, 17B37

Keywords: Representations theory, Hopf algebras, Quantum groups

\section{Introduction}

Hopf algebras theory provides the ring theoretical ground for quantum groups, which has been one of the most active research topics for the past three decades. The discovery of the quantum groups has enriched the classical representations theory of Lie groups. One of the powerful tools in the theory of unitary representations of classical Lie groups is the induction process. Induction of quantum groups representations was developed in several articles, for example $[3,6]$.

From [5, Section 4.1], for a Hopf algebra $H$, the module structure on a left $H$-module $W$, induces a specific left module structure on $H \otimes W$, (given in part (1) of Remark 2.2). $W$ will be a left $H$-Hopf module if it caries a comodule structure that is consistent with the module structure. The consistency 
condition can be phrased as; the comodule structure defines a module homomorphism from $W$ to $H \otimes W$ with respect to the specific module structure on $H \otimes W$, Definition 2.4. Theorem 2.3 provides two other equivalent conditions for the consistency condition. In this work, we relax the consistency condition by freeing it from the specific module structure on $H \otimes W$. A left Hopf representation $W$ is defined to be a left $H$-module that has a comudule structure which defines a homomorphism from $W$ to $H \otimes W$ with respect to some module structure on $H \otimes W$, Definition 2.5. Every Hopf module is by definition a Hopf representation but the converse is not necessarily true, Examples 2.6.

The main result of this article is constructing an induced Hopf representation for a Hopf algebra $H$ using a representation of a quantum subgroup $(B, \pi)$, where $B$ is a bialgebra and $\pi: H \longrightarrow B$ is a bialgebras epimorphism, Theorem 2.12 .

The article is organized as follows. The second section consists of three subsections: first one is devoted for the definition of Hopf modules; in the second subsection we introduce Hopf representations; and in the last subsection we provide the induction construction for Hopf representations. We finish the article with an application on the quantum group $E_{q}(2)$. In the following, let $H=(H, \cdot, 1, \triangle, \epsilon, S)$ be a Hopf algebra over a field $K$ and $W$ be a $K$-space. We will use Sweedler sigma notations, $i d$ as an identity map and $T$ as the twist map.

\section{Hopf Representations}

\subsection{Hopf Modules}

The material of this subsection can be found in $[1,5]$.

Definitions 2.1. $\quad 1 .(W, \alpha)$ is a left $H$-module if the following two diagrams are commutative

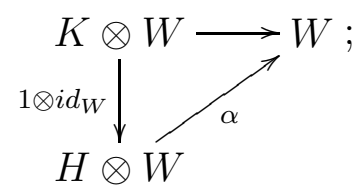

and

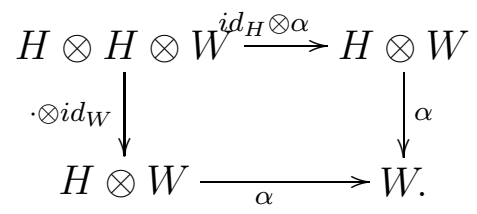


2. $(W, \beta)$ is a left $H$-comodule if the following two diagrams are commutative

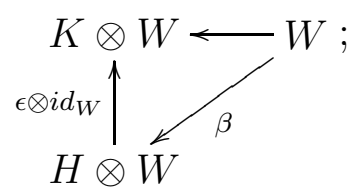

and

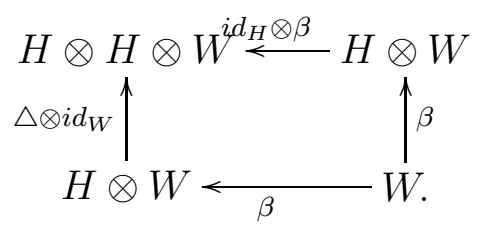

3. Let $\left(W_{1}, \alpha_{1}\right)$ and $\left(W_{2}, \alpha_{2}\right)$ be two left $H$-modules. Then, the map $f$ : $W_{1} \longrightarrow W_{2}$ is called a left $H$-module homomorphism with respect to $\alpha_{1}$ and $\alpha_{2}$ if the following diagram is commutative

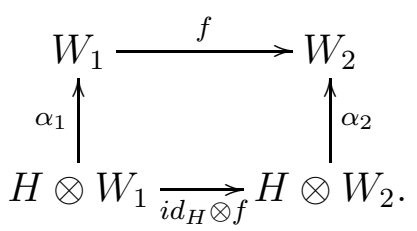

4. Let $\left(W_{1}, \beta_{1}\right)$ and $\left(W_{2}, \beta_{2}\right)$ be two left $H$-comodules. Then, the map $f$ : $W_{1} \longrightarrow W_{2}$ is called a left $H$-comodule homomorphism respect to $\beta_{1}$ and $\beta_{2}$ if the following diagram is commutative

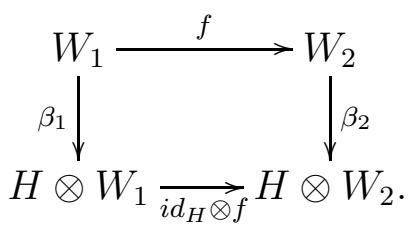

Remark 2.2. 1. If $(W, \alpha)$ is a left $H$-module, then $(H \otimes W, \phi)$ is a left $H$-module with

$$
\phi=(\cdot \otimes \alpha) \circ\left(i d_{H} \otimes T \otimes i d_{W}\right) \circ\left(\triangle \otimes i d_{H \otimes W}\right)
$$

2. If $(W, \beta)$ is a left $H$-comodule, then $(H \otimes W, \psi)$ is a left $H$-comodule with

$$
\psi=\left(\cdot \otimes i d_{H \otimes W}\right) \circ\left(i d_{H} \otimes T \otimes i d_{W}\right) \circ(\triangle \otimes \beta) .
$$


Theorem 2.3. ([1, 5]) Let $(W, \alpha)$ be a left $H$-module and $(W, \beta)$ be a left $H$-comodule. Consider $\phi$ and $\psi$ given in (7) and (8) respectively. Then the following three conditions are equivalent

1. The following diagram is commutative

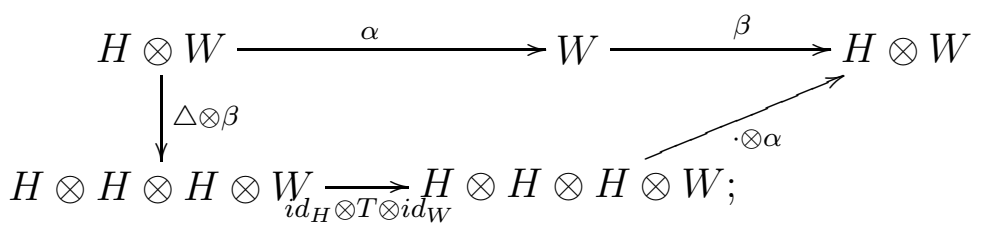

2. $\beta$ is H-module morphism with respect to $\alpha$ and $\phi$;

3. $\alpha$ is $H$-comodule morphism with respect to $\psi$ and $\beta$.

Definition 2.4. The triple $(W, \alpha, \beta)$ is called a left $H$-Hopf module if one the conditions of Theorem 2.3 is satisfied.

\subsection{Hopf Representations}

Definition 2.5. 1. The quadrable $(W, \alpha, \beta, \phi)$ is called a left Hopf representation of the first type if the following three conditions are satisfied

(a) $(W, \alpha)$ is a left $H$ - module;

(b) $(W, \beta)$ is a left $H$-comodule;

(c) $(H \otimes W, \phi)$ is a left $H$-module, such that $\beta$ is $H$-module morphism with respect to $\alpha$ and $\phi$.

2. The quadrable $(W, \alpha, \beta, \psi)$ is called a left Hopf representation of the second type if the following three conditions are satisfied

(a) $(W, \alpha)$ is a left $H$ - module;

(b) $(W, \beta)$ is a left $H$-comodule;

(c) $(H \otimes W, \psi)$ is a left $H$-comodule, such that $\alpha$ is $H$-comodule morphism with respect to to $\beta$ and $\psi$.

3. The quintuple $(W, \alpha, \beta, \phi, \psi)$ is said to be a left $H$-Hopf representation if the following two conditions are satisfied

(a) $(W, \alpha, \beta, \phi)$ is a left Hopf $H$-representation of the first type;

(b) $(W, \alpha, \beta, \psi)$ is a left Hopf $H$-representation of the second type. 
Right Hopf representations can be defined in a similar way with the obvious changes. The second example of the following two examples is a Hopf representation that is not a Hopf module.

Examples 2.6. 1. If $(W, \alpha, \beta)$ is an $H$-Hopf module, then $(W, \alpha, \beta, \phi, \psi)$ is an H-Hopf representation, where $\phi$ and $\psi$ are defined as in (7) and (8) respectively.

2. Let $W=H \otimes H$. Consider the following right $H$-module and $H$-comodule.

$$
\alpha:(H \otimes H) \otimes H \longrightarrow H \otimes H
$$

given by

$$
h \otimes h^{\prime} \otimes h^{\prime \prime} \longmapsto h \otimes h^{\prime} \cdot h^{\prime \prime},
$$

and

$$
\beta: H \otimes H \longrightarrow H \otimes H \otimes H
$$

given by

$$
h \otimes h^{\prime} \longmapsto h_{(1)} \otimes h^{\prime} \otimes h_{(2)} .
$$

One can see that $(W, \alpha)$ is a right $H$-module. To show that $(W, \beta)$ is a right comodule, first for diagram (3) (for right comodules) we have

$$
\begin{aligned}
\left(i d_{H \otimes H} \otimes \epsilon\right) \circ \beta\left(h \otimes h^{\prime}\right) & =\left(i d_{H \otimes H} \otimes \epsilon\right)\left(h_{(1)} \otimes h^{\prime} \otimes h_{(2)}\right) \\
& =h_{(1)} \otimes h^{\prime} \otimes \epsilon\left(h_{(2)}\right) \\
& =h_{(1)} \epsilon\left(h_{(2)}\right) \otimes h^{\prime} \otimes 1 \\
& =h \otimes h^{\prime} \otimes 1 .
\end{aligned}
$$

For diagram (4) (for right comodules) we have

$$
\begin{aligned}
\left(i d_{H \otimes H} \otimes \triangle\right) \circ \beta\left(h \otimes h^{\prime}\right) & =\left(i d_{H \otimes H} \otimes \triangle\right)\left(h_{(1)} \otimes h^{\prime} \otimes h_{(2)}\right) \\
& =h_{(1)} \otimes h^{\prime} \otimes h_{(2)(1)} \otimes h_{(2)(2)} \\
& =h_{(1)(1)} \otimes h^{\prime} \otimes h_{(1)(2)} \otimes h_{(2)} \\
& =\left(\beta \otimes i d_{H}\right) \circ \beta\left(h \otimes h^{\prime}\right) .
\end{aligned}
$$

In the following we will show that $(W, \alpha, \beta)$ is not a right Hopf module by showing that diagram (9) (for right modules and comodules) is not commutative. Let $x$ be a non unit element of $H$. Then

$$
(\beta \circ \alpha)\left(h \otimes h^{\prime} \otimes x\right)=\beta\left(h \otimes h^{\prime} \cdot x\right)=h_{(1)} \otimes h^{\prime} \cdot x \otimes h_{(2)} .
$$

However

$$
(\alpha \otimes \cdot) \circ\left(i d_{H \otimes H} \otimes T \otimes i d_{H}\right) \circ(\beta \otimes \triangle)\left(h \otimes h^{\prime} \otimes x\right)
$$




$$
\begin{aligned}
& =(\alpha \otimes \cdot) \circ\left(i d_{H \otimes H} \otimes T \otimes i d_{H}\right)\left(h_{(1)} \otimes h^{\prime} \otimes h_{(2)} \otimes x_{(1)} \otimes x_{(2)}\right) \\
& =h_{(1)} \otimes h^{\prime} \cdot x_{(1)} \otimes h_{(2)} \cdot x_{(2)} .
\end{aligned}
$$

We define a right Hopf representation structure of the first type on $(W, \alpha, \beta)$. Consider the map

$$
\phi: H \otimes H \otimes H \otimes H \longrightarrow H \otimes H \otimes H
$$

given by

$$
\phi\left(h \otimes h^{\prime} \otimes h^{\prime \prime} \otimes h^{\prime \prime \prime}\right)=h \otimes h^{\prime} \cdot h^{\prime \prime \prime} \otimes h^{\prime \prime} .
$$

To see that $(H \otimes H \otimes H, \phi)$ is an $H$-module. One can see that diagram (1) (for right modules) is commutative for $\phi$, and for diagram (2) (for right modules) we have

$$
\begin{aligned}
\phi \circ\left(\phi \otimes i d_{H}\right)\left(h \otimes h^{\prime} \otimes h^{\prime \prime} \otimes h^{\prime \prime \prime} \otimes \bar{h}\right) & =\phi\left(h \otimes h^{\prime} \cdot h^{\prime \prime \prime} \otimes h^{\prime \prime} \otimes \bar{h}\right) \\
& =h \otimes h^{\prime} \cdot h^{\prime \prime \prime} \cdot \bar{h} \otimes h^{\prime \prime} \\
& =\phi \circ\left(i d_{W} \otimes H \otimes \cdot\right)\left(h \otimes h^{\prime} \otimes h^{\prime \prime} \otimes h^{\prime \prime \prime} \otimes \bar{h}\right) .
\end{aligned}
$$

Finally, $\beta$ is a right module homomorphism with respect to $\alpha$ and $\phi$, since

$$
\begin{aligned}
\beta\left(\alpha\left(h \otimes h^{\prime} \otimes h^{\prime \prime}\right)\right) & =\beta\left(h \otimes h^{\prime} \cdot h^{\prime \prime}\right) \\
& =h_{(1)} \otimes h^{\prime} \cdot h^{\prime \prime} \otimes h_{(2)} \\
& =\phi\left(h_{(1)} \otimes h^{\prime} \otimes h_{(2)} \otimes h^{\prime \prime}\right) \\
& =\phi\left(\beta\left(h \otimes h^{\prime}\right) \otimes h^{\prime \prime}\right) .
\end{aligned}
$$

Which makes $(W, \alpha, \beta, \phi)$ a first type right Hopf representation.

A subspace $W^{\prime}$ of a left (respect to right) Hopf representation $W$ is called a sub Hopf representation of $W$ if the restrictions of the module, comodule and consistency structure maps on $W^{\prime}$ form Hopf representation on $W^{\prime}$. $W$ is called irreducible if it does not have any nontrivial sub Hopf representation. The following two remarks are handy.

Remarks 2.7. 1. The tensor product of two left (respect to right) Hopf representations is a a left (respect to right) Hopf representation.

2. If $W^{\prime}$ is a sub Hopf representation of $W$, then $W / W^{\prime}$ has a unique Hopf representation structure such that the projection map $\rho: W \rightarrow W / W^{\prime}$ is a module and comodule map. 


\subsection{Induced Hopf Representations}

Definition 2.8. ([3],[6]) Let $\left(B,{ }_{B}, 1, \triangle_{B}, \epsilon_{B}\right)$ be a bialgebra. The pair $(B, \pi)$ is called a quantum subgroup of $H$ if $\pi: H \rightarrow B$ is a bialgebra epimorphism, that is $\pi$ is an algebra epimorphism such that the following two diagrams are commutative

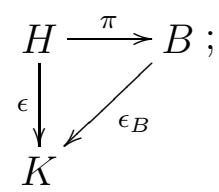

and

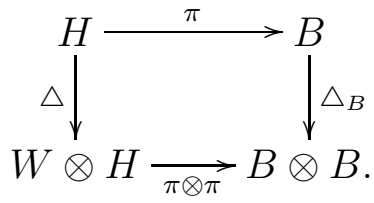

In the following, Let $(B, \pi)$ be a quantum subgroup of $H$ and $(L, \eta)$ be a $B$-module, where $L$ is a Hilbert space.

The space of the induced representation as a subspace of $H \otimes L$ is given by

$$
L^{\prime}=\{h \otimes l ; R(h) \otimes \eta(b \otimes l)=h \otimes 1 \otimes \eta(b \otimes l), \quad \forall b \in B\}
$$

where

$$
R=\left(i d_{H} \otimes \pi\right) \circ \triangle .
$$

The following two lemmas show that $L^{\prime}$ is non trivial.

Lemma 2.9. $1 \otimes l \in L^{\prime}, \quad$ for all $l \in L$.

Proof. Straightforward from the definition of $L^{\prime}$.

Lemma 2.10. If $h \otimes l \in L^{\prime}$ then $h \otimes \eta(b \otimes l) \in L^{\prime}$, for all $b \in B$

Proof. Since $h \otimes l \in L^{\prime}$, then

$$
R(h) \otimes \eta(b \otimes l)=h \otimes 1 \otimes \eta(b \otimes l) .
$$

Now, if $b^{\prime}$ is an element of $B$, then

$$
\left.R(h) \otimes b^{\prime} \otimes \eta(b \otimes l)=h \otimes 1 \otimes b^{\prime} \otimes \eta(b \otimes l)\right) .
$$

Apply the following map on both sides

$$
i d_{H} \otimes i d_{H} \otimes \eta
$$

we get

$$
R(h) \otimes \eta\left(b^{\prime} \otimes \eta(b \otimes l)\right)=h \otimes 1 \otimes \eta\left(b^{\prime} \otimes \eta(b \otimes l)\right) .
$$


Lemma 2.11. If $h \otimes l \in L^{\prime}$, then $\triangle(h) \otimes l \in H \otimes L^{\prime}$.

Proof. Let

$$
f: L \longrightarrow H \otimes L, \quad f(l)=1 \otimes l
$$

Then, we have

$$
\begin{aligned}
\left(i d_{H} \otimes i d_{H} \otimes f\right)(\triangle(h) \otimes l) & =\left(\triangle \otimes i d_{H \otimes L}\right) \circ\left(i d_{H} \otimes f\right)(h \otimes l) \\
& =\left(\triangle \otimes i d_{H \otimes L}\right) \circ\left(R \otimes i d_{L}\right)(h \otimes l) \\
& \left.=\left(\left(\triangle \otimes i d_{H}\right) \circ R\right) \otimes i d_{L}\right)(h \otimes l) \\
& \left.=\left(\left(i d_{H} \otimes R\right) \circ \triangle\right) \otimes i d_{L}\right)(h \otimes l) \\
& =\left(i d_{H} \otimes R \otimes i d_{L}\right)\left(\triangle \otimes i d_{L}\right)(h \otimes l) .
\end{aligned}
$$

Where the second equation is by using $h \otimes l \in L^{\prime}$ with setting $b=1$ in the definition of $L^{\prime}$, and the fourth equation is by using the coassociativity and (20). Now, for $b \in B$, we have

$$
\triangle(h) \otimes 1 \otimes b \otimes l=h_{(1)} \otimes R\left(h_{(2)}\right) \otimes b \otimes l .
$$

Hence

$$
\triangle(h) \otimes 1 \otimes \eta(b \otimes l)=h_{(1)} \otimes R\left(h_{(2)}\right) \otimes \eta(b \otimes l),
$$

which finishes the proof.

Theorem 2.12. $\left(L^{\prime}, \alpha, \beta, \phi_{H}\right)$ is a first type Hopf representation of $H$, with

$$
\begin{gathered}
\alpha: H \otimes L^{\prime} \longrightarrow L^{\prime}, \text { where } \alpha\left(h \otimes h^{\prime} \otimes l\right)=h^{\prime} \otimes \eta(\pi(h) \otimes l), \\
\beta: L^{\prime} \longrightarrow H \otimes L^{\prime}, \text { where } \beta(h \otimes l)=\triangle(h) \otimes l,
\end{gathered}
$$

and the consistency map

$$
\phi_{H}: H \otimes H \otimes L^{\prime} \rightarrow H \otimes L^{\prime}, \quad \text { by } \quad \phi_{H}\left(h \otimes h^{\prime} \otimes h^{\prime \prime} \otimes l\right)=h^{\prime} \otimes h^{\prime \prime} \otimes \eta(\pi(h) \otimes l) .
$$

Proof. First, to see that $\left(L^{\prime}, \alpha\right)$ is a left $H$-module, we have

$$
\begin{aligned}
\alpha \circ\left(1 \otimes i d_{L^{\prime}}\right)(k \otimes h \otimes l) & =\alpha(k \otimes h \otimes l) \\
& =h \otimes \eta(\pi(k) \otimes l) \\
& =k(h \otimes l) .
\end{aligned}
$$

And

$$
\begin{aligned}
\alpha \circ\left(i d_{H} \otimes \alpha\right)\left(h \otimes h^{\prime} \otimes h^{\prime \prime} \otimes l\right) & =\alpha\left(h \otimes h^{\prime \prime} \otimes \eta\left(\pi\left(h^{\prime}\right) \otimes l\right)\right) \\
& =h^{\prime \prime} \otimes \eta\left(\pi(h) \otimes \eta\left(\left(\pi\left(h^{\prime}\right) \otimes l\right)\right)\right. \\
& =h^{\prime \prime} \otimes \eta \circ\left(i d_{H} \otimes \eta\right)\left(\pi(h) \otimes \pi\left(h^{\prime}\right) \otimes l\right) \\
& =h^{\prime \prime} \otimes \eta \circ\left(\cdot_{B} \otimes i d_{L}\right)\left(\pi(h) \otimes \pi\left(h^{\prime}\right) \otimes l\right) \\
& =h^{\prime \prime} \otimes \eta\left(\pi\left(h \cdot h^{\prime}\right) \otimes l\right) \\
& =\alpha \circ\left(\cdot \otimes i d_{L^{\prime}}\right)\left(h \otimes h^{\prime} \otimes h^{\prime \prime} \otimes l\right) .
\end{aligned}
$$


Second, to show that $\left(L^{\prime}, \beta\right)$ is a left $H$-comodule, we have

$$
\begin{aligned}
\left(\epsilon \otimes i d_{L^{\prime}}\right) \circ \beta(h \otimes l) & =\left(\epsilon \otimes i d_{L^{\prime}}\right)(\triangle(h) \otimes l) \\
& =\epsilon\left(h_{(1)}\right) \otimes h_{(2)} \otimes l \\
& =1 \otimes h \otimes l .
\end{aligned}
$$

And

$$
\begin{aligned}
\left(\left(\triangle \otimes i d_{L^{\prime}}\right) \circ \beta\right)(h \otimes l) & =\left(\triangle \otimes i d_{L^{\prime}}\right)\left(h_{(1)} \otimes h_{(2)} \otimes l\right) \\
& =\triangle\left(h_{(1)}\right) \otimes h_{(2)} \otimes l \\
& =h_{(1)} \otimes \triangle\left(h_{(2)}\right) \otimes l \\
& =h_{(1)} \otimes \beta\left(h_{(2)} \otimes l\right) \\
& =\left(i d_{H} \otimes \beta\right) \circ \beta(h \otimes l) .
\end{aligned}
$$

$\left(H \otimes L^{\prime}, \phi_{H}\right)$ is a left $H$-module is straightforward. Finally, to show that $\beta$ is a module map with respect to $\alpha$ and $\phi_{H}$, we have

$$
\begin{aligned}
\beta\left(\alpha\left(h \otimes h^{\prime} \otimes l\right)\right) & =\beta\left(h^{\prime} \otimes \eta(\pi(h) \otimes l)\right) \\
& =\triangle\left(h^{\prime}\right) \otimes \eta(\pi(h) \otimes l) \\
& =\phi_{H}\left(h \otimes h_{(1)}^{\prime} \otimes h_{(2)}^{\prime} \otimes l\right) \\
& =\phi_{H}\left(h \otimes \beta\left(h^{\prime} \otimes l\right)\right) \\
& =\left(\phi_{H} \circ\left(i d_{H} \otimes \beta\right)\right)\left(h \otimes h^{\prime} \otimes l\right) .
\end{aligned}
$$

Hence $\left(L^{\prime}, \alpha, \beta, \phi_{H}\right)$ is a first type left Hopf representations of $H$.

Example 2.13. Consider the quantum group $E_{q}(2)$ (over the field of complex numbers $\mathbb{C}$ ) where the basis of its irreducible representation coincides with the irreducible representation of the quantum group $A\left(E_{q}(2)\right)$, where $A\left(E_{q}(2)\right)$ is the Hopf algebra generated by the elements $z, \bar{z}, a$ and $\bar{a}$ with the commutation relations

$$
z \bar{z}=\bar{z} z=1, a \bar{a}=\bar{a} a, z a=q a z, a z=q \bar{z} a, \bar{a} \bar{z}=q \bar{z} \bar{a}, z \bar{a}=q \bar{a} z
$$

where $q$ is a real number.

The comultiplication is given by

$$
\triangle(z)=z \otimes z, \triangle(a)=a \otimes 1+z \otimes a .
$$

The counit and the antipode are given respectively by

$$
\epsilon(z)=1, \epsilon(a)=0, S(z)=\bar{z}, S(a)=-\bar{z} a .
$$

We can choose a quantum subalgebra $A(K)$ corresponding to translations in $A\left(E_{q}(2)\right)$ which defined as $\mathbb{C}[t, \bar{t}]$. The coproduct $\triangle_{K}$, counit $\epsilon_{K}$ and antipode $S_{K}$ are given by

$$
\triangle_{K}(t)=t \otimes 1+1 \otimes t, \epsilon_{K}(t)=0, S_{K}(t)=-t
$$


and the projection epimorphism, $\pi: A\left(E_{q}\right) \longrightarrow \mathbb{C}[t, \bar{t}]$ is given by $\pi(a)=$ $t, \pi(z)=1$. The Hopf subalgebra $A(K)$ is coabelian and its irreducible corepresentations are one-dimensional. Now, if $\eta$ is the representation of $\mathbb{C}[t, \bar{t}]$ in $\mathbb{C},($ for example $\eta(t \otimes c)=\epsilon(t) c=0$ and $\eta(1 \otimes c)=c$ ) then we have irreducible first type Hopf representation of $A\left(E_{K}(2)\right)$ on the sub Hilbert space $L^{\prime}$.

Acknowledgements. The author was supported by University of Wisconsin Colleges 2014 Summer Research Grant while preparing this work.

\section{References}

[1] A. B. E, Hopf algebra, Cambridge university press, 1980.

[2] J. Dixmier, Enveloping algebras, University Paris, 1977.

[3] A. Gonzalez-Ruiz, L.A. Ibort, Induction of Quantum groups, Phys. Lett. $B, 296$ (1992), 107-110.

[4] S. Majid, Foundation of quantum group theory, Cambridge University press, 1995.

[5] Moss E. Sweedler, Hopf algebras, Cornell University, New York, 1969.

[6] Nicola Ciccoli, Induction of quantum group representation, Journal of Geometry and Physics 31, 2 (1998), 1-16.

\section{Received: February 12, 2014}

